\title{
In Situ technologies and observation strategies for carbon cycling in hadal trenches
}

\author{
FRANK WENZHOEFER ${ }^{1,2,3}$, BO THAMDRUP ${ }^{4}$, \\ KAZUMASA OGURI ${ }^{4}$ AND RONNIE N GLUD ${ }^{4}$ \\ ${ }^{1}$ University of southern Denmark \\ ${ }^{2}$ Alfred-Wegener - Institute \\ ${ }^{3}$ Max Planck Institute for Marine Microbiology \\ ${ }^{4}$ University of Southern Denmark \\ Presenting Author: wenzhoefer@biology.sdu.dk
}

Hadal axis sediments present one of the most extreme habitats on Earth. However, recent investigations have shown that they are deep sea hot spots for benthic mineralization of organic material. Due to the extreme high pressure and low temperature, it is important to assess process rates via in situ measurements. Therefore, we have developed and combined a suite of autonomous instrumentation to allow measurements of food supply, benthic processes, and benthic species composition and abundance. This presentation will describe new hadal instrumentation and observation strategies for monitoring of biogeochemical processes and communities with the high spatial and temporal resolution necessary to evaluate their contribution to hadal processes.

Three autonomous benthic lander systems are used to estimate biogeochemical processes, like benthic oxygen uptake, denitrification, anammox and sulfate reduction in situ. HadalProfiler and Hadal-Chamber Lander are used to determine the benthic oxygen consumption as a proxy for carbon degradation. The Hadal-Profiler Lander, equipped with a transecting microprofiler capable to resolve multiple vertical sets of oxygen concentration profiles across the sediment-water interface along a horizontal distance of $70 \mathrm{~cm}$, providing data to quantify the diffusive oxygen uptake. The Hadal-Chamber Lander, incubating a certain area of seafloor, is used to measure total solute exchange rates at the benthic interface including all relevant transport processes (diffusion, advection and fauna-mediated transport). This lander will also be used to perform in situ experiments at the trench bottom; isotopically labelled phytodetrital material will be injected into the chambers to follow the benthic turn over and the importance of the different functional groups. The Hadal-Sediment Lander can retrieve intact sediment cores from great depth, and additionally allows in situ tracer incubations by injecting, e.g., isotopically labelled nitrate, nitrite or ammonia to study the benthic nitrogen cycle. To resolve and understand short- and long-term deposition dynamics, a mooring system combines sediment traps with current and CTD measurements and an imaging system. These time-lapse cameras, mounted also on all landers, allow to monitor sediment structure, benthic fauna, sedimentation and sediment processes during the respective deployments. Using this multidisciplinary, concerted and quantitative approach we will gain new knowledge on hadal ecosystems. 